\title{
Safety and efficacy of surgical fixation of fibula fractures using an intramedullary nail: a retrospective observational cohort study in 30 patients
}

Guilherme Boni ${ }^{1,2,3}$, Gustavo T. Sanchez ${ }^{1}$, Gustavo Arliani ${ }^{1}$, Boris A. Zelle ${ }^{4^{*}}$, Robinson E. Pires ${ }^{5}$ and Fernando B. dos Reis ${ }^{1,3}$

\begin{abstract}
Background: Open reduction and internal fixation remains the standard treatment for displaced unstable ankle fractures. Plate fixation represents the most frequently used instrumentation option in fibula fractures and favourable outcomes have been reported. Recently, intramedullary nailing techniques have been suggested as a viable alternative resulting in less soft tissue disruption. The objectives of this study are to describe the surgical technique and to evaluate the safety and efficacy of using an intramedullary nail in patients undergoing surgical fixation of their fibula fracture.
\end{abstract}

Methods: A total of 30 skeletally mature patients with unstable ankle fracture who underwent intramedullary fixation of their fibula fractures from February 2016 to July 2017 were included in this retrospective study. Patients were evaluated using the Short Form-36 (SF-36) and the American Orthopaedic Foot and Ankle Society (AOFAS) at 18 months after surgery.

Results: All patients went on to fracture union. Two patients required a secondary surgical procedure. No patient included in this series developed any wound complications. The mean Physical Component Summary (PCS) of the SF-36 was $53.90 \pm 13.3$ and the mean Mental Component Summary Score (MCS) was $52.63 \pm 11.12$. The AOFAS subscale scores were $34.67 \pm 1.03$ for pain, $42.40 \pm 0.2997$ for function and $9.50 \pm 0.2785$ for alignment.

Conclusions: Our study demonstrates promising outcomes associated with intramedullary nail fixation of unstable fibula fractures. We recommend intramedullary nail fixation of fibula fractures to be a safe procedure with a low complication rate.

Level of evidence: Level 4 retrospective case series.

Keywords: Fibula fracture, Intramedullary fixation, Patient safety; quality of life

\section{Background}

Ankle fractures account for approximately $9 \%$ of all fractures and up to $22 \%$ of all lower limb fractures $[1,2]$. While many ankle fractures can be treated non-operatively, open reduction and internal fixation remains the standard treatment for unstable and displaced ankle fractures [1]. The primary objectives of surgical treatment are to restore the

\footnotetext{
* Correspondence: zelle@uthscsa.edu

${ }^{4}$ Department of Orthopaedics, University of Texas Health Science Center at San Antonio, 7703 Floyd Curl Dr, MC-7774, San Antonio, TX 78229, USA Full list of author information is available at the end of the article
}

ankle anatomy and allow for early mobilization. Internal plate fixation remains the most commonly used instrumentation option in the treatment of unstable fibula fractures [3]. Despite favourable outcomes associated with the use of plate fixation, the potential risks of wound dehiscence, infection, and implant failure, continue to represent areas of concern, in particular in patients with diabetes, elderly patients, and patients with significant soft tissue injuries [3, 4]. In addition, hardware prominence remains a common complaint and frequently requires implant removal $[5,6]$. In order to address these potential 
shortcomings of fibula plate fixation, intramedullary nailing has been explored as an alternative treatment option [7]. In particular, patients with significant injuries to the surrounding soft tissue envelope may potentially benefit from this technique [8]. Despite these potential advantages, the safety and efficacy of intramedullary nail fixation of fibula fractures requires further investigation.

The objectives of this study are to describe the surgical technique and to evaluate the safety and efficacy of intramedullary nail fixation of fibula fractures. We hypothesize that intramedullary nail fixation will be associated with a relatively low rate of secondary surgical procedures as compared to traditional fixation methods reported in the literature.

\section{Methods}

This retrospective study was approved by the Institutional Review Board (IRB) of the Federal University of São Paulo (protocol 1,880,524). All patients participating in this study were provided full disclosure and written informed consent was obtained from all subjects enrolled.

Patients with unstable ankle fractures treated February 2016 and July 2017 were included in this retrospective study. The inclusion criteria for enrolment in this study included skeletally mature patients with unstable fibula fractures (Danis-Weber [9] type B and type C) treated with an intramedullary nail. Fracture instability was determined by the treating surgeon, based on incongruency of the mortise, medial clear space widening, syndesmosis widening, or significant fracture displacement. Skeletally immature patients as well as patients, who were unwilling to provide consent, were excluded from this study. All patients were operated on by the same team of board-certified orthopaedic surgeons. In all patients, surgical fixation of the fibula was performed with an intramedullary locked reamed nail (Acumed $^{\circ}$ solid titanium rod, Hillsboro, OR, United States).

\section{Surgical technique}

The surgery is performed in the supine position on a standard radiolucent operating room Table. A minimalinvasive reduction is performed using an approximately 1-cm lateral incision directly over the fracture site in order to allow for direct visualization of the fracture reduction. The fracture reduction is maintained by application of a percutaneously placed pointed reduction clamp. A small stab incision is made over the distal tip of the fibula. The intramedullary canal is accessed using a $6.1-\mathrm{mm}$ cannulated opening reamer, which is placed over a $1.6-\mathrm{mm}$ guidewire. The diaphysis is reamed with a $3.1-\mathrm{mm}$ or $3.7-\mathrm{mm}$ cannulated reamer to prepare the canal for stem placement. The implant is available in two diameters $(3 \mathrm{~mm}$ and $3.6 \mathrm{~mm}$ ) and three lengths $(110 \mathrm{~mm}, 145 \mathrm{~mm}$ and $180 \mathrm{~mm})$. Associated syndesmotic injuries can be addressed by placement of syndesmotic screws inserted through the nail in a percutaneous fashion. Two anterior to posterior interlocking screws and two lateral to medial interlocking screws are used to lock the nail distal and proximal to the fracture (Fig. 1).

Our postoperative treatment protocol includes toetouch weight bearing immediately after surgery and initiation of early range of motion exercises. At 4 weeks after surgery, patients are allowed to initiate weight bearing as tolerated to the injured lower extremity. At 2 months after surgery, patients are cleared for all activities of daily living. Patients are allowed to return to sports at 3months postoperatively.

\section{Collection of outcome data}

Clinical and radiographic follow-up data were recorded at subsequent routine follow-up appointments up until 18 months after surgery. Standardized postoperative radiographs including anteroposterior, lateral, and mortise views were obtained during the follow-up visits in order to evaluate fracture healing, nail position, and maintained fracture reduction. The main outcome measure was need for secondary surgical procedures. Secondary outcome measures included surgical site complications, such as non-union, loss of reduction, hardware loosening, wound dehiscence, infections, peroneal tendon injuries, or peri-implant fractures. Fracture union was defined as presence of bridging callus on three out of four cortices on anteroposterior and lateral views. Additional secondary outcome measures included the functional outcomes at 18 months following surgery as measured by the Short Form-36 (SF-36) [10] as well as the American Orthopaedic Foot and Ankle Society (AOFAS) score [11]. The SF-36 is a widely-used standardized scoring system for measuring the health-related quality of life. It has been validated and in various languages and has been used in numerous clinical orthopaedic and non-orthopaedic studies [12]. The SF-36 allows for calculation of eight subscales including physical functioning, role physical, bodily pain, general health, vitality, social functioning, role emotional, mental health. The results can be summarized in the Physical Component Summary (PCS) measure and the Mental Component Summary (MCS) measure. The scores range between 0 and 100 with higher scores reflecting superior quality of life. The AOFAS was used as a foot and ankle specific outcome measure. This clinical rating system includes both patient-reported outcomes as well as provider-reported outcomes based on a physical examination assessing motion, stability, and alignment. The AOFAS can be divided into three subscales pain, function and alignment. The maximum score for each of these subscales is 40 points for pain, 50 points for function, and 10 points for alignment. The AOFAS sum scores range from 0 points 


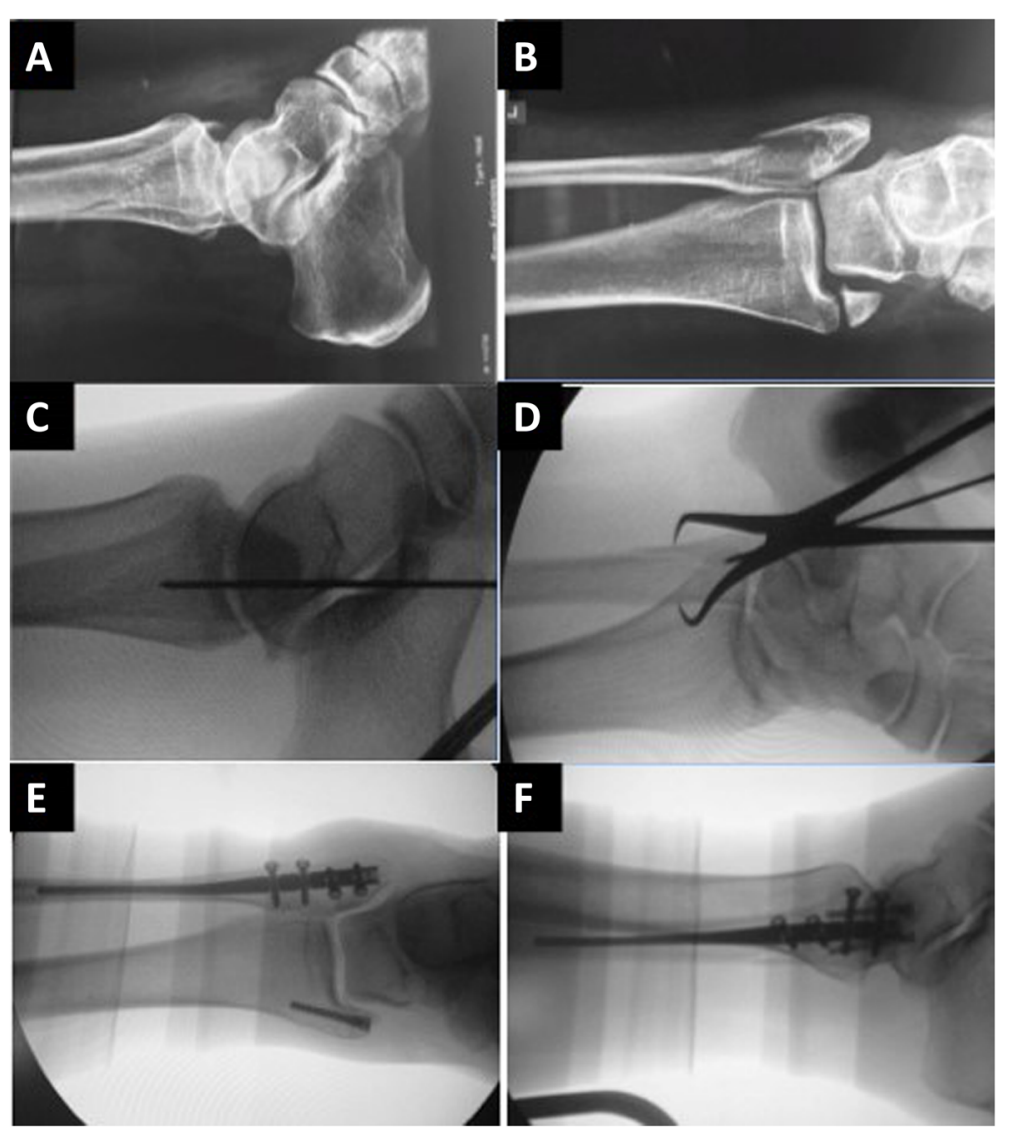

Fig. 1 Lateral and anteroposterior radiographs of the ankle in a patient with a displaced bimalleolar ankle fracture (1A-B). Intraoperative fluoroscopic images showing the passage of the guidewire at the tip of the lateral malleolus and reduction with a percutaneous reduction forceps (1C-D). Fluoroscopic images documenting acceptable fracture reduction and appropriate implant position (1E-F)

(maximum impairment) to 100 points (no impairment). The AOFAS has undergone multiple validation studies and remains one of the most widely-used foot and ankle specific outcome measures [13].

\section{Statistical analysis}

We calculated the the AOFAS and SF-36 score using the standard scoring algorithms for the two questionnaires. The database was maintained in Microsoft Excel 2010 sheets (Microsoft, Redmond, WA). All continuous variables were tested for normal distribution using the Kolmogorov-Smirnov test. We expressed data as means, standard deviations and percentages. We used the used SPSS 22.0 for Mac software in the analysis.

\section{Results}

A total of 30 patients (12 female, 18 male), with unstable fibula fractures were surgically treated using this fixation method. All patients were operated on within $24 \mathrm{~h}$ of their injury. The mean age of patients was $50.5 \pm 12.61$ years. Danis-Weber type B fractures accounted for $70 \%$ $(n=21)$ and Danis-Weber type $C$ fracture accounted for
$30 \%(n=9)$ of all fractures. A total of 17 fractures (56.7\%) presented as lateral malleolus fractures and 13 fractures (43.3\%) presented as bimalleolar fractures.

\section{Outcomes}

All patients included in this series completed their follow-up and no patients were lost to follow-up. Regarding our main outcome measure, two patients required a secondary surgical procedure. The first patient experienced a significant loss of reduction, because he started playing volleyball at 7 days after surgery, and the radiographs showed a loss of reduction with $10^{\circ}$ of valgus malalignment. The second patient required a revision surgery due to a new injury during the postoperative period. This patient suffered a fall of the ladder on the twelfth day after surgery resulting in new peri-implant fracture around the distal portion of the nail. In this patient, the fibula nail was converted to a plate fixation construct and the fibula fracture ultimately healed uneventfully.

Regarding our secondary outcome measures, nonunions, hardware loosening, wound dehiscences, infections, peroneal tendon injuries were not observed in this study. 
Two patients suffered loss of reduction less than $2 \mathrm{~mm}$. This was noticed at the two-months and 3 months followup appointments, respectively. As both patients remained asymptomatic, a revision surgery was not deemed to be necessary by the treating surgeon. Overall, patients achieved favourable SF-36 scores with a mean Physical Component Summary (PCS) of $53.90 \pm 13.3$ and a mean Mental Component Summary Score (MCS) of $52.63 \pm$ 11.12. Table 1 shows the results of the eight SF-36 subscales. The AOFAS subscale scores were $34.67 \pm 1.03$ for pain, $42.40 \pm 0.2997$ for function and $9.50 \pm 0.2785$ for alignment. The results of the AOFAS scales are demonstrated in Table 2.

\section{Discussion}

Unstable ankle fractures are commonly treated by open reduction and internal fixation, using techniques that can be associated with several complications including, loss of reduction, wound healing problems, and infections. In addition, several common symptoms include pain, stiffness, swelling, and instability of the ankle joint [14]. As many as $17-24 \%$ of patients may experience unsatisfactory outcomes [15]. The use of an intramedullary fibular nail represents has been suggested as a feasible alternative to plate fixation with the potential to reduce surgical site complications [16]. Our study data suggests that the use of a fibular nail in patients with unstable ankle fractures is associated with a low complication rate and satisfactory clinical outcomes. We suggest that intramedullary nail fixation of unstable ankle fractures represents a safe surgical procedure.

The outcomes achieved in our patient series compare favorably with previously reported studies. Obremskey et al. [17] reported on 20 Weber type B and C ankle fractures treated with plate fixation and recorded an overall SF-36 score of 71.56 at 20 months post-surgery. In an observational study, Bhandari et al. [18] reported on 30 patients with unstable ankle fractures undergoing fibula plate fixation. These authors recorded a mean SF36 score of 69.78 at 24 months after surgery. Moreover,
Table 2 AOFAS subscales and sum score at 18 months followup

\begin{tabular}{lllll}
\hline AOFAS & Maximum & Minimum & Mean & $\begin{array}{l}\text { Standard } \\
\text { deviation }\end{array}$ \\
\hline Pain & 40 & 20 & 34.67 & 1.03 \\
Function & 50 & 34 & 42.401 & 0.2997 \\
Alignment & 10 & 8 & 9.500 & 0.2785 \\
\hline
\end{tabular}

the average physical function score at 20 and 24 months was 64.7 and 67.5 , respectively. In this context, the SF36 scores recorded in our study suggest very favorable functional outcomes that can be achieved using this fibula fixation technique. We were able to demonstrate a low rate of secondary surgical procedures and high patient satisfaction scores using standardized and validated health-related quality of life scores, which allow for appropriate comparisons with other patient populations [19]. Surprisingly, some of the SF-36 scales in our series trended even higher than in the normative patient population. The safety data recorded in this study are also in line with recent publications reporting relatively low complication rates associated with the use of a locked intramedullary fibular nail [20-23].

Our study has both strengths and limitations. We suggest that a strength of our study is the single center experience with a homogenous patient population and a standardized surgical and rehabilitation protocol. In addition, we were able to record appropriate follow-up information as all patients enrolled in this study completed their follow-up. Limitations of our study include its retrospective design. In addition, we do not have a comparison group from our center and we can only compare our results with data from the literature. In addition, we report on a relatively small sample size and the exact infection rates may require further investigation in larger series and meta-analyses of the available literature. Future studies may further delineate the specific indications for intramedullary nail fixation of the fibula versus other traditional fixation techniques.

Table 1 SF-36 scores at 18 months following surgery

\begin{tabular}{llllll}
\hline Domains & Maximum & Minimum & Mean Score & Standard deviation & $\begin{array}{c}\text { Mean } \\
\text { Normative Data }\end{array}$ \\
\hline Physical Function & 100 & 65 & 79.34 & 20.03 & 82.45 \\
Role Physical & 100 & 76 & 88.34 & 21.50 & 74.73 \\
Pain & 97 & 84 & 82.43 & 14.89 & 67.53 \\
General health & 95 & 88 & 86.56 & 8.37 & 71.10 \\
Vitality & 100 & 79 & 81.66 & 19.79 & 66.85 \\
Social aspects & 100 & 72 & 88.75 & 19.75 & 78.30 \\
Role Emotional & 94 & 79 & 84.40 & 15.83 & 70.02 \\
Mental health & 100 & 92 & 89.99 & 6.44 & 73.82 \\
\hline
\end{tabular}




\section{Conclusion}

Our results demonstrate the potential of intramedullary nail fixation as a safe method for surgical treatment of unstable fibula fractures. The results of our study suggest promising clinical and functional outcomes as well as low complication rates.

\section{Abbreviations}

AOFAS: American Orthopaedic Foot and Ankle Society; MCS: Mental Component Summary; PCS: Physical Component Summary; SF-36: Short Form-36

\section{Acknowledgements}

The publication costs for this article (APC) were covered in full by a grant from the Colorado Physician Insurance Company (www.copic.com). COPIC had no influence on authorship or scientific content of this article.

\section{Ethics approval and consent participate}

This study was approved by the Institutional Review Boards of the Federal University of São Paulo.

\section{Authors' contributions}

GB: Made substantial contributions to conception and design, acquisition of data, data analysis, interpretation of data, and preparation of manuscript. GTS: Made substantial contributions to conception and design, acquisition of data, and final manuscript review. GA: Made substantial contributions to conception and design, acquisition of data, and final manuscript review. BAZ: Made substantial contributions to data analysis, interpretation of data, and preparation of manuscript. REP: Made substantial contributions to conception and design, acquisition of data, and final manuscript review. FBR: Made substantial contributions to conception and design, interpretation of data, and preparation of manuscript. All authors have read and approved the final data.

\section{Funding}

The publication costs for this article were covered in full by a grant from the Colorado Physician Insurance Company (www.copic.com) to Philip F. Stahel, MD. COPIC had no influence on authorship or scientific content of this article. There were no additional sources of funding obtained for this study.

\section{Availability of data and materials}

The datasets generated and/or analyzed during the current study are not publicly available due the presence of personal health information but available from the corresponding author on reasonable request.

\section{Consent for publication}

There is no information with individual personal data so no consent for publication was needed.

\section{Competing interests}

BAZ has received speaker fees from AO North America, consultant fees from KCI USA, Inc., research grants from Department of Defense, DepuySynthes, $\mathrm{KCl}$ USA, Inc., Orthopaedic Trauma Association, and educational grants from the Center for Orthopaedic Trauma Advancement and Smith and Nephew Inc. He serves on the editorial board of International Orthopaedics, Journal of Bone and Joint Surgery, Journal of Orthopaedic Trauma, and Patients Safety in Surgery. None of these activities are related to the content of this article. The remaining authors declare that they have no competing interests.

\section{Author details}

'Department of Orthopaedics and Traumatology, Federal University of São Paulo, São Paulo, SP, Brazil. ${ }^{2}$ IFOR - Instituto de Fraturas, Ortopedia e Reabilitação, São Paulo, SP, Brazil. ${ }^{3}$ HSC - Hospital São Camilo, São Paulo, SP, Brazil. ${ }^{4}$ Department of Orthopaedics, University of Texas Health Science Center at San Antonio, 7703 Floyd Curl Dr, MC-7774, San Antonio, TX 78229, USA. ${ }^{5}$ Department of the Locomotor Apparatus, Federal University of Minas Gerais, Belo Horizonte, MG, Brazil.
Received: 27 June 2019 Accepted: 24 September 2019

Published online: 15 October 2019

\section{References}

1. Rockwood \& Green Fractures in Adults. - 7 Edition : 2010: 1975-2003.

2. Court-Brown CM, Caesar B. Epidemiology of adult fractures: a review. Injury. 2006;37:691-7.

3. Lynde MJ, Sautter T, Hamilton GA, Schuberth JM. Complications after open reduction and internal fixation of ankle fratures in the elderly. Foot Ankle Surg. 2012;18:103-7.

4. Linchfield JC. The treatment of unstable fractures of the ankle in the elderly. Injury. 1987;18:128-32

5. Sanders DW, Tieszer C, Corbett B. Operative versus nonoperative treatment of unstable lateral malleolar fratures: a randomized multicenter trial. J Orthop Trauma. 2012;32(26):129-34.

6. Ribeiro de Ávila V, Bento T, Gomes W, Leitão J, Fortuna de Sousa N. Functional outcomes and quality of life after ankle fracture surgically treated: A systematic review. J Sport Rehabil. 2018;27:274-83.

7. Asloum Y, Bedin B, Roger T, Charissoux JL, Arnaud JP, Mabit C. Internal fixation of the fibula in ankle fractures: a prospective, randomized and comparative study: plating versus nailing. Orthop Traumatol Surg Res. 2014;100(4 Suppl): S255-9. https://doi.org/10.1016/j.otsr.2014.03.005 Epub 2014 Apr 4.

8. Lain S, Haughton BA, Brew C. Intramedullary fixation of distal fibular fractures: a systematic review of clinical and functional outcomes. J Orthop Traumatol. 2014;15:245-54

9. Court-Brown CM, Heckman JD, McQueen MM, et al. Rockwood and Green's - fractures in adults, 8th edition. Philadelphia, PA 2015; 2547-2556.

10. Ware JE, Sherbourne CD. The MOS 36-item short-form health survey (SF-36). I. Conceptual framework and item selection. Med Care. 1992;30(6):473-83.

11. Kitaoka HB, Alexander IJ, Adelaar RS, Nunley JA, Myerson MS, Sanders M. Clinical rating sistems for the ankle-hindfoot, mid-foot, hallux and lesser toes. Foot Ankle Int. 1994;15:135-49.

12. Patel AA, Donegan D, Albert T. The 36-item short form. J Am Acad Orthop Surg. 2007;15(2):126-34.

13. Hunt KJ, Hurwit D. Use of patient-reported outcome measures in foot and ankle research. J Bone Joint Surg Am. 2013; 95(16):e118(1-9).

14. Bjørslev N, Ebskov L, Lind M, Mersø C. High complication rate after surgical treatment of ankle fractures. Ugeskr Laeger. 2014;176(32):1494-6.

15. Lee $Y S$, Huang HL, Lo TY, Huang CR. Lateral fixation of $A O$ type-B2 ankle fractures in the elderly: the Knowles pin versus the plate. Int Orthop. 2007;31:817-21.

16. Clarín KE, Bugler KE, Watson CD, Hardie AR, Appleton P, McQueen MM, Court-Brown CM, White TO. The treatment of unstable fractures of the ankle using the Acumed fibular nail: development of a technique. J Bone Joint Surg (Br). 2012:94(8):1107-12

17. Obremeskey WT, Dirschl DR, Crowther JD, Craig WL, Driver RE, LeCroy CM Change over time of SF-36 functional outcomes for operatively treated unstable ankle fractures. J Orthop Trauma. 16(1):30-3.

18. Bhandari M, Sprague S, Hanson B, Busse JW, Dawe DE, Moro JK, Guyatt GH. Health-related quality of life following operative treatment of unstable ankle fractures: a prospective observational study. J Orthop Trauma. 2004;18(6):338-45.

19. SooHoo NF, Vyas R, Samimi D. Responsiveness of the foot function index, AOFAS clinical rating systems, and SF-36 after foot and ankle surgery. Foot Ankle Int. 2006;27(11):930-4

20. Asloum Y, Bedin B, Roger T, Charissoux JL, Arnaud JP, Mabit C. Internal fixation of the fibula in ankle fractures: a prospective, randomized and comparative study: plating versus nailing. Orthop Traumatol Surg Res. 2014; 100(4 Suppl):S255-9.

21. Carter TH, Mackenzie SP, Bell KR, Bugler KE, MacDonald D, Duckworth AD, White TO. Optimizing long-term outcomes and avoiding failure with the fibula intramedullary nail. J Orthop Trauma. 2019;33(4):189-95.

22. Jordan RW, Chapman AWP, Buchanan D, Makrides P. The role of intramedullary fixation in ankle fractures - a systematic review. Foot Ankle Surg. 2018;24(1):1-10.

23. White TO, Bugler KE, Appleton P, Will E, McQueen MM, Court-Brown CM. A prospective randomised controlled trial of the fibular nail versus standard open reduction and internal fixation for fixation of ankle fractures in elderly patients. Bone Joint J. 2016;98-B(9):1248-52

\section{Publisher's Note}

Springer Nature remains neutral with regard to jurisdictional claims in published maps and institutional affiliations. 